\title{
D2D Resource Allocation Algorithm in Heterogeneous Networks
}

\author{
Ting $\mathrm{YU}^{1}$, Liang $\mathrm{YE}^{1,2} *$ \& Xuejun SHA ${ }^{1}$ \\ ${ }^{1}$ Harbin Institute of Technology, Harbin, Heilongjiang Province, China
}

Esko Alasaarela ${ }^{2}$

${ }^{2}$ University of Oulu, Oulu, Finland

\begin{abstract}
Device-to-device (D2D) communication has recently emerged as a promising technique to improve the performance of LTE-A systems. It can greatly reduce the load of the base station, improve the quality of service for users at the edge of the cell, and improve the overall throughput in the system. After analyzing the resource allocation method of the SFR model, we come to a conclusion that this method is suitable for the reuse of D2D cellular resources. We establish the system model, and propose a resource allocation algorithm. The simulation results show that using the proposed algorithm, D2D communication can improve the system throughput.
\end{abstract}

KEYWORD: D2D; SFR; resource allocation; throughput

\section{INSTRUCTION}

In recent years, with the development of mobile communication and the increasing demand for system capacity, the existing $3 \mathrm{G}$ technology becomes unable to meet the increasing mobile broadband business requirements. At the same time, the $4 \mathrm{G}$ mobile communication that can adapt to the mobile data, mobile computing and mobile multimedia operation began to rise. The $4 \mathrm{G}$ communication technology is another evolution of wireless communication technology after the $3 \mathrm{G}$ mobile communication. It was developed to improve the access rate of mobile terminals to the Internet. However, the physical layer and the MAC layer which use effective 4G cellular technology (WiMAX[1] and LTE-A[4]) still can't meet the explosive data requirement of mobile users. So we need to find a revolutionary approach to change the traditional cellular network. Device-to-Device (D2D) is likely to appear in the next generation of cellular technology methods.

D2D communication in the cellular network is defined as two mobile users those can communicate directly without the base station (BS) or core networks. D2D communication is not often transparent for cellular networks. It can use either the in-band spectrum or the out-band spectrum. In traditional cellular networks, all the communications need to be controlled by the BS, even when the communication distance is very close, i.e. within the scope of the D2D communication, communications also need a BS. The architecture of cellular networks is fit for traditional low data rate mobile communications, such as voice communication and text messages, where the distance between the communication users is not close enough to communicate directly. However, in today's cellular network, mobile users may use high data rate services (such as video sharing, games and social network) within the scope of direct communication (D2D). Therefore, in this scenario, D2D can improve the spectrum efficiency of the network. The D2D communication advantages can not only improve the efficiency of frequency spectrums, but also increase the system throughput, improve energy utilization efficiency, reduce the time delay, and increase the fairness of the system.

In [7], the author proposed a resource allocation method that reused uplink spectrum resources and avoided interference between cellular and D2D communication. The core idea of this method was: by testing the public control channel, the base station could find cellular users with high risk of near-far interfere. The base station could sign these cellular users and broadcast the information. Based on the information, D2D users could choose resources actively to avoid heavy interference from other users. In [2], the author first used a power control method to control the D2D communication interference within a certain range, and then studied a control strategy to reduce the interference which the cellular communication caused to the D2D communication. The author put forward a concept of 
Interference Limited Area (ILA), defined it as the area where the ISR of D2D receiving user is larger than the threshold, and proposed an interference managing method based on ILA control. This method did not allow D2D users to reuse spectrum resources within the ILA to manage interference. Reference [5] proposed a similar method to the reference [2]. The author proposed an ILA interference managing method based on distance limit. It considered the resource sharing of a pair of D2D users on the edge of the cellular. The author thought that in the uplink, the distance between two D2D users was much closer than that between a D2D transmitting user and the base station, so the D2D communication interference to the cellular could be negligible. To avoid the large disturbance caused by cellular to the D2D communication, the author argued that when the distance between D2D receiver and cellular users was greater than a certain threshold, D2D users could reuse the cellular spectrum resources.

\section{RESOURCE ALLOCATION IN THE CELL}

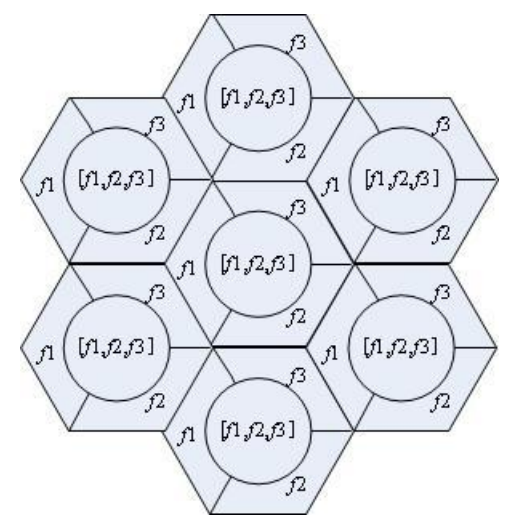

Figure 1. SFR frequency allocation

LTE uses the Soft Frequency Reuse (SFR) resource allocation model to solve the interference problem of the traditional allocation model and the FFR model where the spectrum utilization rate is not high [6]. As shown in Fig. 1, the entire cell frequency is divided into three parts: $f 1, f 2$ and $f 3$. In the SFR model, center cell users use all frequency resources, and each sector of the edge users uses one out of the three frequencies. Let the edges of the neighboring cells use different spectrum. As in the center cell, the cellular users' distance is close to the BS, and the BS is far from users at the edge of the cell, so we can set the transmit power of cellular users in edge cell twice larger than that in the center cell. So the receiving power is almost the same. The distance between D2D users is close, so the interference from D2D users to cellular users or the BS is small. As shown in Fig. 1, when the D2D user is at the edge of the $\mathrm{S} 3$ sector, $f 1$ and $f 2$ resources can be allocated to him/her. When the D2D user is at the edge of the cell, the receiving power from $\mathrm{D} 2 \mathrm{D}$ transmitter to the BS is very low. When the cellular users in the center cell transmit to the BS, the interference to the $\mathrm{D} 2 \mathrm{D}$ receiver is very low. There is no same frequency interference from the cellular users at the edge of cell to D2D users. This model can improve the cell spectrum efficiency and the system throughput.

\section{SYSTEM MODEL}

There are $I$ cellular users and $J$ pairs of D2D users in the heterogeneous system [3]. The set of cellular users is $\left\{\mathrm{CUE}_{1}, \mathrm{CUE}_{2}, \cdots, \mathrm{CUE}_{i}, \cdots, \mathrm{CUE}_{I}\right\}$, and the set of D2D users is $\left\{\mathrm{D} 2 \mathrm{D}_{1}, \mathrm{D} 2 \mathrm{D}_{2}, \cdots\right.$, $\left.\mathrm{D} 2 \mathrm{D}_{j}, \cdots, \mathrm{D} 2 \mathrm{D}_{J}\right\}$. Each pair of the D2D users consists of a transmitter and a receiver. D2D users reuse the uplink resources of cellular network. Define the reuse matrix as $X=\left[x_{i, j}\right]$, where

$x_{i . j}= \begin{cases}1 & \text { if } \mathrm{D}_{2} \mathrm{D}_{\mathrm{j}} \text { reuses } \mathrm{CUE}_{\mathrm{i}} \\ 0 & \text { others }\end{cases}$

Assume that $p_{i}$ is the transmit power of $\mathrm{CUE}_{i}$, thus the receiving SINR of the $i^{\text {th }}$ uplink is

$$
\beta_{i}^{c}=\frac{p_{i} \cdot g_{i, B S}}{\sigma^{2}+\sum_{j \in J} x_{i, j} \cdot I N_{i, c}^{j}}
$$

where $g_{i, B S}$ is the channel gain between $\mathrm{CUE}_{i}$ and the BS. $I N_{i, c}^{j}$ is the potential interference from receiver $\mathrm{D}_{2} \mathrm{D}_{j}$ to the uplink receiver of $\mathrm{CUE}_{i}$ (i.e. BS). $\sigma^{2}$ is the noise power. In the real system, the target SINR of the cellular link should meet the Quality-of-Service (QoS) requirement. Different cellular uplink service needs different SINR. Assume that $\beta_{i, t g t}^{c}$ is the target SINR which $\mathrm{CUE}_{i}$ needs. In order to guarantee the priority of the cellular uplink, we need to make sure that each uplink receiving SINR is higher than the threshold, that is

$\beta_{i}^{c} \geq \beta_{i, \text { tgt }}^{c}, \forall i \in I$

Assume that $p_{i}$ is the transmit power of $\mathrm{D} 2 \mathrm{D}_{j}$, thus the receiving SINR of the $j^{\text {th }}$ uplink is

$$
\beta_{j}^{d}=\frac{p_{i} \cdot g_{j, j}}{\sigma^{2}+\sum_{n \in N} \sum_{j \in I} x_{i, j} \cdot I N_{j, d}^{j}}
$$

where $g_{j, j}$ is the channel gain from transmitting to receiving of $\mathrm{D} 2 \mathrm{D}_{j} . \quad I N_{j, d}^{j}$ is the potential interference from the uplink receiver of $\mathrm{CUE}_{i}$ to receiver $\mathrm{D}_{2} \mathrm{D}_{j}$. The simulation is in multi-cell, so we need to consider the interference among different cells. We add the interference from neighbor cell 
$\mathrm{CUE}_{i}$ to $\mathrm{D}_{2} \mathrm{D}_{j}$ into the potential interference. When the receiving SINR is lower than threshold, the D2D link may be unreliable. In this case, we can't use this D2D link to communicate. So we set a receiving SINR threshold $\beta_{0}^{d}$, and the reliable D2D link should correspond to the formula

$\beta_{j}^{d} \geq \beta_{0}^{d}, \forall j \in J$

In order to meet cellular communicating requirement in (2), the maximum interference from D2D link to $\mathrm{CUE}_{i}$ link can be described as

$$
I N_{i, c}^{j}=p_{i} \cdot g_{i, B S} / \beta_{i, t g t}^{c}-\sigma^{2}
$$

When the $\mathrm{D}_{2} \mathrm{D}_{j}$ link reuses the resource of $\mathrm{CUE}_{i}$ link, the interference added in $\mathrm{CUE}_{i}$ cellular link can be expressed as $p_{j} \cdot g_{j, B S}$. The transmit power of $\mathrm{D}_{2} \mathrm{D}_{j}$

$$
p_{j} \leq \min \left\{p_{j}^{\max }, I N_{i, c} / g_{j . B S}\right\}
$$

where $p_{j}^{\max }$ is the maximum of D2D transmit power. According to the (3) and (4), in order to guarantee the reliability of the D2D link, the transmit power

$$
p_{j} \geq \beta_{0}^{d} \cdot\left(\sigma^{2}+\sum_{n \in N} I N_{j, d}^{i}\right) / g_{j, j}
$$

At the same time considering (6) and (7), we can know when $D 2 D_{j}$ and $C_{U} E_{i}$ share the same resources, D2D communication transmission power

$$
\begin{aligned}
& p_{j} \in P_{j}(i), \\
& P_{j}(i)=\left\{p \mid \beta_{0}^{d} \cdot\left(\sigma^{2}+\sum_{n \in N} I N_{j, d}^{i}\right) / g_{j, j} \leq p\right. \\
& \left.\leq \min \left\{p_{j}^{\max }, I N_{i, c} / g_{j, B S}\right\}\right\}
\end{aligned}
$$

$P_{j}(i)=\varnothing$ means the $\mathrm{D}_{2} \mathrm{D}_{j}$ link is not allowed to reuse $\mathrm{CUE}_{i}$ link resources. $P_{j}(i) \neq \varnothing$ means the $\mathrm{D} 2 \mathrm{D}_{j}$ link is allowed to reuse $\mathrm{CUE}_{i}$ link resources. Define a $I \times J$ allowable reuse matrix $A=\left[a_{i, j}\right]$, where

$a_{i, j}= \begin{cases}1, & P_{j}(i) \neq \varnothing \\ 0, & P_{j}(i)=\varnothing\end{cases}$

D2D transmit power should correspond to (8). In order to reduce power consumption and the D2D communication interference, the D2D transmit power can be chosen as its minimum value, that is $p_{j}=\beta_{0}^{d} \cdot\left(\sigma^{2}+\sum_{n \in N} I N_{j, d}^{i}\right) / g_{j, j}$. The interference from $\mathrm{D} 2 \mathrm{D}_{j}$ transmitter to $\mathrm{BS}$ is $I N_{i, c}^{j}=p_{j} \cdot g_{j, B S}$. Define interference matrix $B=\left[b_{i, j}\right]$. When $a_{i, j}=0, b_{i, j}$ is infinity. When $a_{i, j}=1, b_{i, j}=I N_{i, c}^{j}$. The optimization problem can be described as

$$
\min \sum_{i \in I} \sum_{j \in J} \mathrm{~b}_{i, j} \cdot x_{i, j}
$$

$$
\text { s.t. } \begin{cases}(i) & \sum_{i \in I} x_{i, j}=1, \forall j \in J \\ \text { (ii) } & \sum_{j \in J} x_{i, j} \leq 1, \forall i \in I \\ \text { (iii) } \quad & x_{i, j} \in\{0,1\}\end{cases}
$$

Limitation ( $i$ ) ensures that each D2D link can reuse the resources of a cellular link. Limitation ( $i i)$ guarantees that each cellular link resource is reused by at most one D2D link. Limitation (iii) means the constraint variables $x_{i, j}$ can only be 0 or 1 .

\section{RESOURCE ALLOCATION ALGORITHM}

The idea of the proposed resource allocation algorithm is: first choose the minimum value from the $I \times J$ interference matrix. Then allocate the corresponding value of the resources to the corresponding D2D users. Search the minimum interference value in the matrix which consists of unallocated D2D users and un-reused cellular users, and allocate the resources. Repeat this step, and if the interference value is greater than the threshold, the D2D user returns to the original interference matrix to be allocated.

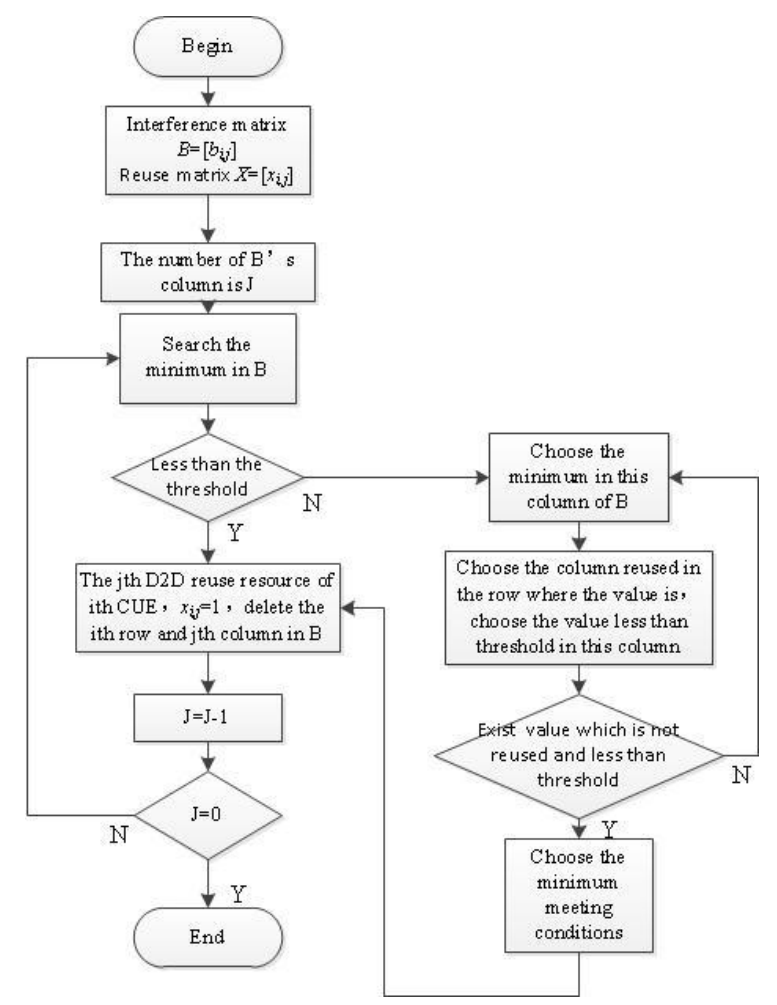

Figure 2. Algorithm flow chart

\section{SIMULATION RESULTS}

In the system level simulation, we first allocate resources to the cellular users. After all the cellular users have been allocated with resources, use the algorithm in Section 4 to allocate resources to D2D users. This algorithm can make D2D users reuse 
high QoS spectrum resources and improve the throughput of the system.

In the simulation of this section, we mainly observe the situation in which the D2D communication users are contained in cellular communication system. As the number of D2D users varies, the throughput of the cellular communication system changes. It provides support for practical system design. The number of cellular users is fixed to 63 in the simulation, and change the number of D2D users. Observe the changes of the overall system throughput. The simulation results are shown in Fig. 3 and Fig. 4.

From Fig. 3 and Fig. 4, we can see that as the number of D2D users increases, the throughput of cellular users decrease slowly, and the overall throughput of D2D users increases. So the overall throughput of system increases. In terms of average throughput of each user, as the number of the D2D users increases, the cellular user's average throughput falls slightly, and the D2D user's average throughput also falls slightly. But the system average throughput increases. The reason is that the D2D users are at the edge of the cell, the distance to the $\mathrm{BS}$ is far, and transmitting power of D2D users is low, so it has little impact to the BS. The distance of each D2D user pair is close, and the distance between D2D users and cellular users is far. The interference on D2D users by cellular users is small. So each D2D user's average throughput is large. As the number of D2D users increases, the multiplexing channel quality declines, so the D2D average throughput decreases.

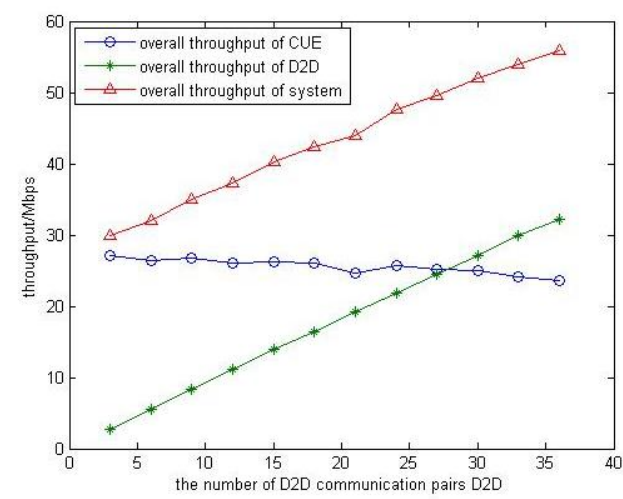

Figure 3. Overall throughput

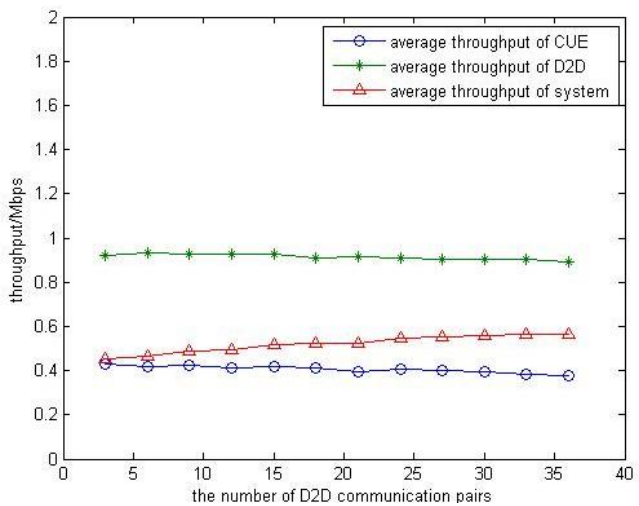

Figure 4. Average throughput

\section{CONCLUSION}

This paper introduced the characteristics of the D2D communication and discussed that D2D communication in the cellular system can help to improve the performance of the system. After analyzing the SFR model resource allocation method, we proved that this method is suitable for D2D reusing cellular resources. We established the system model, and proposed a resource allocation algorithm. The simulation results showed that using the proposed algorithm, D2D communication can improve the system overall throughput.

\section{ACKNOWLEDGEMENTS}

This work was supported by the Natural Scientific Research Innovation Foundation in Harbin Institute of Technology under Grant No. HIT.NSRIF. 2013029, the National Science and Technology Major Project under Grant No. 2012ZX03004003, the 973 Program under Grant No. 2013CB329003, and partly by the National Science Foundation of China under Grant No. 61101123.

\section{REFERENCES}

[1] IEEE Standard 802.16e-2005. part16: Air interface for fixed and mobile broadband wireless access systems amendment for physical and medium access control layers for combined fixed and mobile operation in licensed band, 2005.

[2] Min, H. Lee, J. Park, S. Hong, D. 2011. Capacity enhancement using an interference limited area for Deviceto-Device uplink underlaying cellular networks. IEEE Transactions on Wireless Communications, 2011, 10(12): $3995-4000$.

[3] Son, D.J. Yu, C.H. \& Kim, D.I. 2014. Resource Allocation based on Clustering for D2D Communications in Underlaying Cellular Networks. Information and Communication Technology Convergence (ICTC), 2014 International Conference on. pp.232,237, 22-24 Oct. 2014.

[4] Third Generation Partnership Project (3GPP). Physical layer procedures (Release 10) for Evolved Universal Terrestrial Radio Access (EUTRA), 3GPP TS $36.213 \mathrm{v}$ 10.5.0, 2012.

[5] Wang, H. \& Chu, X. 2012. Distance-constrained resourcesharing criteria for Device-to-Device communications underlaying cellular networks. Electronics Letters, 2012, 48(9): $528-530$.

[6] Wu, W. \& Zhang, Y. 2014. Dedicated Resource Allocation for D2D Communications in Cellular Systems Employing FFR. 2014 Sixth International Conference on Wireless Communications and Signal Processing.

[7] Xu, S. Wang, H. Chen, T. Huang, Q. Peng, T. 2010. Effective interference cancellation schemefor Device-toDevice communication underlaying cellular networks. In IEEE 72nd Vehicular Technology Conference Fall (VTC 2010-Fall). 2010, 1 -5. 